\title{
Dynamic Precipitation in a Binary Mg-Al Alloy During Equal Channel Angular Extrusion (ECAE)
}

\author{
Xiaolong $\mathrm{Ma}^{1}$, Suhas Eswarappa-Prameela ${ }^{1}$, Nicholas Krywopusk ${ }^{1}$, Laszlo J. Kecskes ${ }^{2}$, Tomoko Sano ${ }^{3}$ \\ and Timothy P. Weihs ${ }^{1}$ \\ 1. Department of Materials Science and Engineering, Johns Hopkins University, Baltimore, MD USA \\ 2. MATSYS, Inc., 45490 Ruritan Circle, Sterling, VA USA \\ 3. WMRD, US Army Research Laboratory, 4600 Deer Creek Loop, Aberdeen, MD USA
}

Precipitation hardening is one of most effective ways to strengthen magnesium alloys [1]. Conventional static aging in $\mathrm{Mg}-\mathrm{Al}$ alloys produces precipitates which are dominated by high aspect-ratio plates or laths that inhabit the basal planes of $\alpha-\mathrm{Mg}$ crystals. These precipitates are believed to be ineffective at blocking basal slips during deformation, and thus result in little strengthening [1]. The highly anisotropic precipitate morphology is closely pertinent to the distinctive interface energies of various atomic planes in the hexagonal-close-packed structure, which govern the nucleation and growth of precipitates under static conditions.

In this talk, we present a dynamic precipitation phenomenon in a $\mathrm{Mg}$-Al alloy during equal channel angular extrusion (ECAE) at relatively low temperature. To avoid the interference of insoluble manganese in interpreting precipitation behavior [2], we select a solution-treated binary $\mathrm{Mg}-\mathrm{Al}$ alloy for this study. We will emphasize on understanding the microstructure evolution of dynamic precipitation using electron microscopy and comparing it with precipitate characters in conventional aging process. The dynamic precipitation process leads to a bimodal microstructure that includes 1) the original matrix grains containing a high density of finely spaced, continuous $\mathrm{Mg}_{17} \mathrm{Al}_{12}$ precipitates with low aspect ratios and 2) sub-micron sized, recrystallized matrix grains with similarly sized $\mathrm{Mg}_{17} \mathrm{Al}_{12}$ precipitates, as shown in Figure 1a and b. Compared to static aging precipitation, the latter phase is unique and its volume fraction increases with the number of ECAE passes. In addition, the widely-observed cellular discontinuous precipitates in static aging is not seen [3]. In terms of similarity, the continuous precipitates and the original matrix grains maintain the same orientation relationship of $(0001)_{\mathrm{Mg}} / /(011)_{\text {precipitate }}$ (see Figure 1c) as the case in static aging [4] but this is not true for recrystallized phase.

More importantly, using X-ray diffraction and energy dispersive spectroscopy (EDS) under TEM, it is found, for the first time, that the completeness of precipitation process is distinctive in two microstructures. The depletion of Al in original matrix grains is significantly less than that in the recrystallized matrix grains (see Figure $2 a$ and $b$ ), indicating a higher level of precipitation completeness in the recrystallized phase. The implication is that the precipitation process may occurs simultaneously with recrystallization within those local regions. This is also consistent with the observed solute concentration (only 1 2a.t.\% Al) in the recrystallized magnesium grains, which is close to the level on equilibrium phase diagram under the processing temperature. The sub-micro sized precipitates are nucleated from the both high-angle grain boundaries $\left(>15^{\circ}\right)$ in the originally material and newly generated low-angle grain boundaries with localized strain and energy (Figure 3).

These results provide insights into understanding dynamic precipitation behaviour in magnesium alloys. 
References:

[1] J.F. Nie, Metallurgical and Materials Transactions A 43 (2012) p.3891-3939.

[2] J. Gubicza et al, Journal of Alloys and Compounds 492 (2010) p.166-172.

[3] J. Clark, Acta Metallurgica 16 (1968) p.141-152

[4] D. Duly et al, Philosophical Magazine A 71 (1995) p.187-204.

[5] The authors acknowledge funding from U.S. Army Research Laboratory for its financial support through the Materials in Extreme Dynamic Environments award W911NF-12-20022.
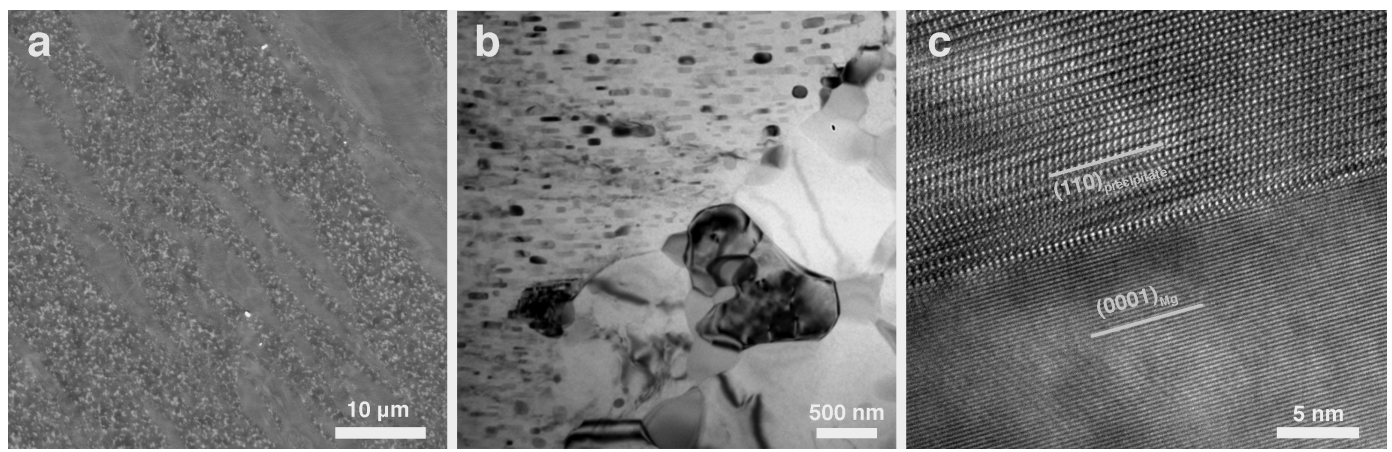

Figure 1. (a) Backscattering electron SEM micrograph of a representative bi-modal microstructure of ECAE-processed Mg-9wt\%Al alloy; (b) Bright-field TEM micrograph of the interfacial region between two distinctive microstructures; (c) High-resolution TEM of interfacial structure between precipitate and matrix grains in the original coarse-grained region.

a

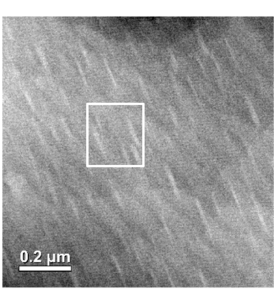

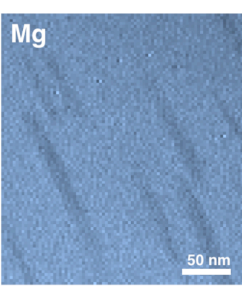

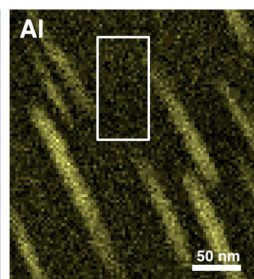

b

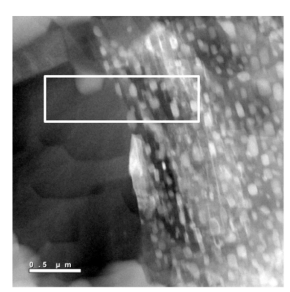

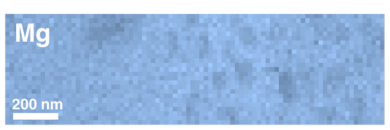

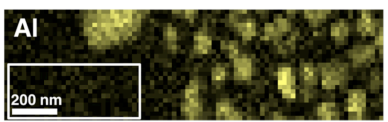

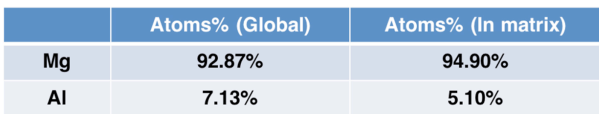

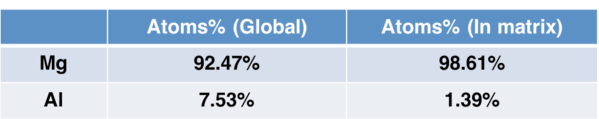

Figure 2. EDS mapping of a precipitated region in (a) an original matrix grain; (b) recrystallized region; and the corresponding quantifications in the magnesium matrix.
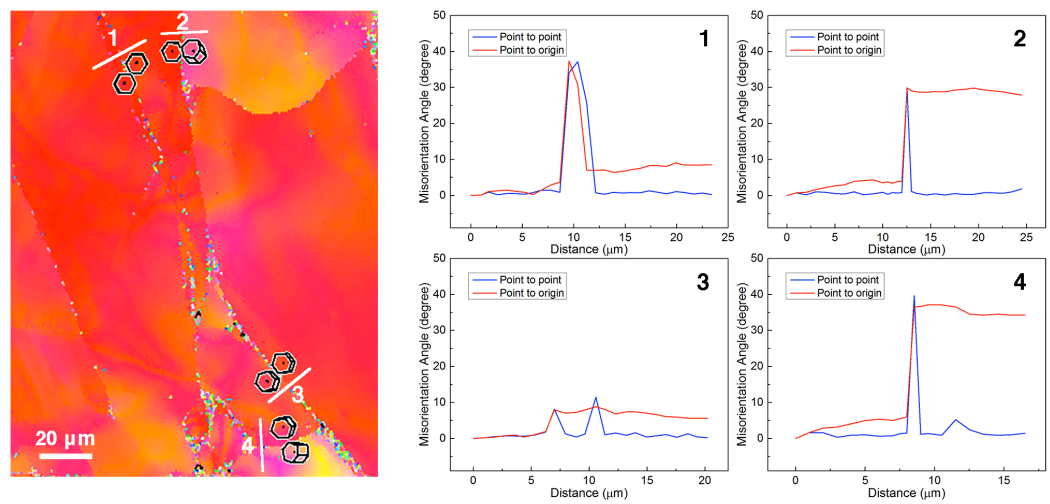

Figure 3. EBSD mapping of a typical region where recrystallized precipitates are seen and the corresponding misorientation angle profiles in the number-marked grain boundaries. 